\title{
Ontogeny of the Pre-Bötzinger Complex in Perinatal Rats
}

\author{
Silvia Pagliardini, Jun Ren, and John J. Greer \\ Department of Physiology, Centre for Neuroscience, University of Alberta, Edmonton, Alberta, Canada T6G 2S2
}

The aim of this study was to provide a systematic examination of the ontogenesis of the mammalian respiratory rhythm generating center, the pre-Bötzinger complex (pre-BötC). A combination of immunohistochemical markers and electrophysiological recordings was used to determine the time of inception of the pre-BötC and the developmental changes during the perinatal period in rats spanning from embryonic day 15 (E15) to postnatal day 7. The first clear indication of neurons immunopositive for neurokinin-1 receptors (NK1Rs) and somatostatin expression, two proposed markers for pre-BötC neurons, was at $\sim$ E17. Birth dating of neurons in the ventrolateral medulla using 5-bromo-2'-deoxyuridine demonstrated that NK1R-positive neurons populating the area of the pre-BötC during late E16 -E18 are born at E12.5-E13.5, $\sim 2 \mathrm{~d}$ later than adjacent NK1R-positive neurons in the ventrolateral medulla. Extracellular recordings of neuronal populations within the pre-BötC of perinatal medullary slice preparations demonstrated that the onset of rhythmical respiratory discharge commences at $\sim$ E17. Application of substance P, a ligand for NK1R receptors, to the media bathing E17 medullary slice and brainstem-spinal cord preparations resulted in a marked increase in respiratory frequency. These data provide insights into the ontogeny of the pre-BötC, giving fundamental information on the genesis, settlement, and inception of rhythmic activity within the group of neurons proposed to be responsible for the respiratory rhythm generation. Furthermore, this provides the foundation for further analyses of cell lineage, the transcriptional control of respiratory neuronal development, and electrophysiological and pharmacological properties of the pre-BötC during the prenatal period.

Key words: pre-Bötzinger complex; breathing; perinatal; medulla; respiration; neurokinin-1 receptor

\section{Introduction}

The pre-Bötzinger complex (pre-BötC) is composed of a group of neurons located in the ventrolateral medulla that are essential for the generation of the mammalian respiratory rhythm (for review, see Rekling and Feldman, 1998; Feldman et al., 2003). Given the functional importance of this region, it has become a major focus of research in the field of respiratory neural control. This study is the first to examine the ontogeny of the pre-BötC. Immunohistochemical and electrophysiological techniques were used to discern when the pre-BötC forms and how its anatomical organization and rhythmic neuronal activity change during the perinatal period in rats. Collectively, these data provide the necessary foundation for understanding critical stages of mammalian respiratory center development.

The pre-BötC, originally identified in a seminal study published by Smith et al. (1991), is located ventral to the semicompact division of the nucleus ambiguus (NA), caudal to the compact division of the NA and rostral to the lateral reticular nucleus (LRN) formation. Subsequent experiments using electrophysiological recordings, pharmacological manipulations, and lesions, both in vitro and in vivo, confirmed that the pre-BötC is critically

Received May 15, 2003; revised Aug. 18, 2003; accepted Aug. 22, $2003 .$.

This work was supported by the Canadian Institutes of Health Research (CIHR) and the Alberta Lung Association. J.J.G. is a scientist of the Alberta Heritage Foundation for Medical Research (AHFMR). S.P. and J.R. are recipients of an AHFMR studentship and a CIHR Alberta Sudden Infant Death Syndrome Foundation fellowship, respectively. We thank Dr. Wei Zhang and Dr. Ba Fang for technical assistance.

Correspondence should be addressed to Dr. John J. Greer, Department of Physiology, Centre for Neuroscience, University of Alberta, 513 Heritage Medical Research Centre Building, Edmonton, Alberta, Canada T6G 2S2. E-mail: john.greer@ualberta.ca.

Copyright $\odot 2003$ Society for Neuroscience $\quad$ 0270-6474/03/239575-10\$15.00/0 involved in the generation of the respiratory rhythm (Smith et al., 1991; Ramirez et al., 1998; Solomon et al., 1999; Gray et al., 2001; Sun et al., 2001).

Gray et al. (1999) proposed that pre-BötC neurons could be identified by immunolabeling for neurokinin-1 receptors (NK1Rs). Although demonstrating that NK1R-immunopositive neurons were also present outside the pre-BötC, subsequent studies supported the idea that there is a population of small fusiform neurons expressing NK1Rs in the pre-BötC that have characteristics consistent with their being involved in respiratory rhythmogenesis (Pilowsky and Feldman, 2001; Wang et al., 2001; Guyenet et al., 2002). Further support came from experiments demonstrating that bilateral destruction of NK1R-expressing neurons within the pre-BötC by substance $\mathrm{P}(\mathrm{SP})$ conjugated to saporin results in ataxic breathing (Gray et al., 2001). Collectively, the data indicates that NK1R immunolabeling is a useful tool for demarcating the proposed region underlying respiratory rhythmogenesis and thus was used in this study. Recent evidence suggests that somatostatin (SST) immunolabeling may also be a marker of neurons within the pre-BötC (Stornetta et al., 2003) and thus was incorporated into this study.

5-Bromo-2' deoxyuridine (BrdU) labeling was used to examine the birth dates of respiratory nuclei in the medulla. If NK1Rpositive neurons within the pre-BötC neurons are truly distinct, as hypothesized, then we expected this to be reflected by differences in the relative birth dates of respiratory neurons within the ventrolateral medulla.

Recordings from spinal ventral roots of embryonic brainstem-spinal cord preparations had demonstrated that respiratory discharge commenced within the cervical spinal cord at em- 
bryonic day 17 (E17) in the rat (Greer et al., 1992; DiPasquale et al., 1996). Confirmative data were derived from ultrasound recordings of fetal breathing movements (FBMs) in utero from unanesthetized dams (Kobayashi et al., 2001). However, those data demonstrate the commencement of inspiratory drive transmission within spinal motoneuron populations. Whether this reflected the onset of activity within the pre-BötC is unknown and therefore is addressed in the current study.

\section{Materials and Methods}

Animal handling. Sprague Dawley rats were bred at the University of Alberta and all procedures were approved by the Animal Welfare Committee of the institution. The timing of pregnancies was determined from the appearance of sperm plugs in the breeding cages, and the embryonic age of rats was confirmed by measuring crown-rump length (Angulo y González, 1932). E0 was considered the day of vaginal plug detection. Fetal rats were delivered from timed pregnant rats anesthetized with halothane (1.25-1.5\% delivered in $95 \% \mathrm{O}_{2}$ and $5 \% \mathrm{CO}_{2}$ ) and maintained at $37^{\circ} \mathrm{C}$ by radiant heat. Newborn rats were anesthetized by inhalation of metofane $(2-3 \%)$.

Immunohistochemistry. We examined the distribution of NK1R-, SST-, and choline acetyl transferase (ChAT)-immunoreactive neurons within the ventrolateral medulla from ages E15 to postnatal day 7 (P7). The rabbit polyclonal antibody to rat NK1R (residues 393-407) was previously characterized (Vigna et al., 1994; Mantyh et al., 1997). Transverse and sagittal sections $(50 \mu \mathrm{m})$ were serially collected and immunoreacted. Single- and double-labeling experiments for NK1R (Advanced Targeting Systems, San Diego, CA), ChAT (Chemicon, Temecula, CA), and SST (Immunostar, Hudson, WI) were performed on free-floating sections according to the following protocol. Sections were rinsed in PBS and incubated with $1.0 \%$ bovine serum albumin (BSA; Sigma, St. Louis, $\mathrm{MO}$ ) and $0.3 \%$ Triton X-100 in PBS for 60 min to reduce nonspecific staining and to increase antibody penetration. Sections were incubated overnight with primary antibodies (rabbit polyclonal anti-NK1R and anti-SST, diluted 1:1000; goat polyclonal anti-ChAT, diluted 1:400) diluted in PBS containing 0.1\% BSA and 0.3\% Triton X-100. After several washes in PBS, sections were incubated with secondary antibodies conjugated to the fluorescent probes $\mathrm{Cy} 3$-conjugated donkey anti-rabbit (Cy3-DAR, 1:200; Jackson ImmunoResearch, West Groove, PA) and Cy5-conjugated donkey anti-goat (Cy5-DAG, 1:200; Jackson ImmunoResearch) diluted in PBS and $0.1 \%$ BSA for $2 \mathrm{hr}$. Sections were further washed in PBS and mounted and coverslipped with Fluorsave mounting medium (Calbiochem, San Diego, CA). In a subset of experiments, sections were labeled using a peroxidase method. After overnight incubation with primary antibodies and several washes in PBS, sections were incubated with biotinylated secondary antibodies (bDAR, 1:200; Jackson ImmunoResearch; biotinylated anti-goat, 1:200; Sigma) for $2 \mathrm{hr}$, washed in PBS, and then incubated with standard peroxidase-conjugated ABC (1:100, Vector Laboratories, Toronto, Ontario, Canada). The reaction was then detected with $0.08 \% \mathrm{DAB}$ and $0.007 \% \mathrm{H}_{2} \mathrm{O}_{2}$ in Tris- $\mathrm{HCl}$ buffer. Adjacent sections were counterstained with Thionin (1\%) to visualize the cytoarchitecture of the tissue.

To detect very low expression of NK1R, serial sagittal sections were immunoreacted according to the tyramide signal amplification (TSA) protocol. Briefly, sections were pretreated in $3 \% \mathrm{H}_{2} \mathrm{O}_{2}$ in PBS, washed in TNT buffer ( $0.1 \mathrm{~m}$ Tris-HCl, pH 7.5, $0.15 \mathrm{M} \mathrm{NaCl}$, and $0.05 \%$ Tween 20 ) and incubated in TNB-T $(0.1 \mathrm{~m}$ Tris- $\mathrm{HCl}, \mathrm{pH} 7.5,0.15 \mathrm{M} \mathrm{NaCl}, 0.5 \%$ blocking reagent, and $0.3 \%$ Triton X-100). Sections were incubated with NK1R antibody diluted 1:30,000 in TNB-T overnight. With this dilution, no signal for NK1R receptor-like immunoreactivity was detected unless amplified with the TSA kit. After several washes in TNT, sections were incubated for $2 \mathrm{hr}$ in biotinylated DAR (1:200). Biotin was revealed using the TSA kit (PerkinElmer Life Sciences, Boston, MA). Sections were incubated with Streptavidin-HRP (1:150) for $30 \mathrm{~min}$ followed by tyramide conjugated to fluorescein $(1: 75)$ for $10 \mathrm{~min}$, diluted in amplification diluent.

If sections were immunoreacted for NK1R and SST, the tyramide amplification system was used. After NK1R immunolabeling, the sections were extensively washed in TNT and incubated for $30 \mathrm{~min}$ in TNB-T followed by anti-SST antibody (1:1000) in TNB-T overnight. The following day, sections were incubated with DAR-Cy3 (1:200) in TNB for $2 \mathrm{hr}$, washed, mounted on slides, and coverslipped with Fluorsave solution.

Double and triple labeling for ChAT, NK1R, and BrdU were performed on tissue obtained from embryonic and postnatal rats that had been previously treated with BrdU. Sections were pretreated with $2 \mathrm{~N} \mathrm{HCl}$ for $60 \mathrm{~min}$ to separate the double-stranded DNA and to allow interaction of the primary anti-BrdU antibody with incorporated BrdU. After the acid treatment, the $\mathrm{pH}$ was restored using $0.1 \mathrm{M} \mathrm{Na}_{2} \mathrm{~B}_{4} \mathrm{O}_{7}$ for $10 \mathrm{~min}$, and the sections were incubated with $1.0 \%$ BSA and $0.3 \%$ Triton X-100 in PBS. A mixture of primary antibodies (anti-NK1R, 1:1000; anti-ChAT, 1:400; and mouse monoclonal anti-BrdU, 1:100; BD Biosciences, San Jose, CA) diluted in PBS with $0.1 \%$ BSA and $0.3 \%$ Triton X-100 was applied overnight. Sections were then washed with PBS and incubated with a mixture of fluorescent or biotin-conjugated secondary antibodies diluted 1:200 in 0.1\% BSA and PBS [Cy5-DAG, Cy3-DAR, and biotinylated donkey anti-mouse (bDAM; Jackson ImmunoResearch)]. After a 2 $\mathrm{hr}$ incubation, sections were washed and incubated with streptavidin488 (1:200; Molecular Probes, Eugene, OR) in 0.1\% BSA and PBS for a further $2 \mathrm{hr}$.

Neuronal birth date assessment via BrdU injections. Single pulses of BrdU (Roche Molecular Biochemicals, Indianapolis, IN; $50 \mathrm{mg} / \mathrm{kg}$ of maternal body weight) were injected intraperitoneally into pregnant rats on different gestational ages from E9.5 to E16.5 (at 12 P.M.). BrdUtreated and control pregnant rats or postnatal (P0-P7) rats were anesthetized with halothane or metofane, and the embryos or pups were perfused with phosphate buffer (PB) followed by a solution of $4 \%$ formaldehyde (Sigma) in PB. Brainstems were dissected and postfixed in the same fixative solution, and the tissue was either embedded in agar and cut on a vibrating microtome (VT1000S; Leica, Nussloch, Germany) for single and double labeling or dehydrated, embedded in paraffin, and cut on a microtome ( $8 \mu \mathrm{m}$ thickness) for cell counting.

Confocal imaging. Immunostained sections were scanned with a Zeiss (Oberkochen, Germany) Axioplan microscope (10, 20, or 40×, 1.3 oil objective) using a Zeiss LSM 510 NLO laser configured to a computer running LSM 510 software. For FITC and Alexafluor 488 fluorescence, excitation (argon, $40 \mathrm{mV}$ ) was set to $488 \mathrm{~nm}$, and emissions were collected with a $505 \mathrm{~nm}$ long-pass filter. For Cy3 fluorescence, excitation (HeNe, $1 \mathrm{mV}$ ) was set to $543 \mathrm{~nm}$, and emissions were collected using a $560 \mathrm{~nm}$ long-pass filter. For Cy5 fluorescence, excitation (HeNe, $1 \mathrm{mV}$ ) was set to $633 \mathrm{~nm}$, and emissions were collected using a $630 \mathrm{~nm}$ long-pass filter. Thin sections and multiple sectioning acquisitions along the $z$-plane were performed to obtain a better signal through the depth of the section. Acquired images were then exported in JPEG format, and brightness and contrast were adjusted in Photoshop 6.0 (Adobe Systems, Mountain View, CA).

Cell counting and measurements. Paraffin transverse sections from P7 rats were double-immunolabeled for NK1R and BrdU according to the following protocol. Sections were dewaxed in xylenes and rehydrated in descending alcohols, and antigene retrieval was performed in citrate buffer $(0.01 \mathrm{M} \mathrm{Na}$ citrate, $\mathrm{pH}$ 6.0; Sigma) in a microwave oven $(750 \mathrm{~W})$ for $5 \mathrm{~min}$. Sections were pretreated in $2 \mathrm{~N} \mathrm{HCl}$ as described above for the fluorescent triple-labeling staining protocol, and a mixture of the two primary antibodies was applied overnight (anti-NK1R, diluted 1:700; anti-BrdU, diluted 1:75). The following day, slides were washed in PBS, and sections were incubated with alkaline phosphatase-conjugated goat anti-rabbit antibody (1:50; Sigma) diluted in PBS and 0.1\% BSA for $3 \mathrm{hr}$. NK1R immunoreactivity was then revealed with fast red substrate (Sigma). Sections were extensively washed and incubated with bDAM (1: $200)$ and standard ABC $(1: 100)$ in PBS and $0.1 \%$ BSA. BrdU staining was revealed with $0.08 \% \mathrm{DAB}, 0.05 \%$ nickel ammonium sulfate, and $0.007 \%$ $\mathrm{H}_{2} \mathrm{O}_{2}$. Sections were then washed, dehydrated, and coverslipped.

Three to four animals per BrdU treatment were analyzed. Sections from the caudal end of the facial nucleus (VII) to the rostral end of the LRN were immunoreacted. One in every four sections in the pre-BötC area was captured with a Nikon (Melville, NY) 990 digital camera mounted on a Leica DMRBE microscope with a $20 \times$ objective. NK1Rpositive neurons in a $400 \times 540 \mu \mathrm{m}$ box, centered below the NA and 

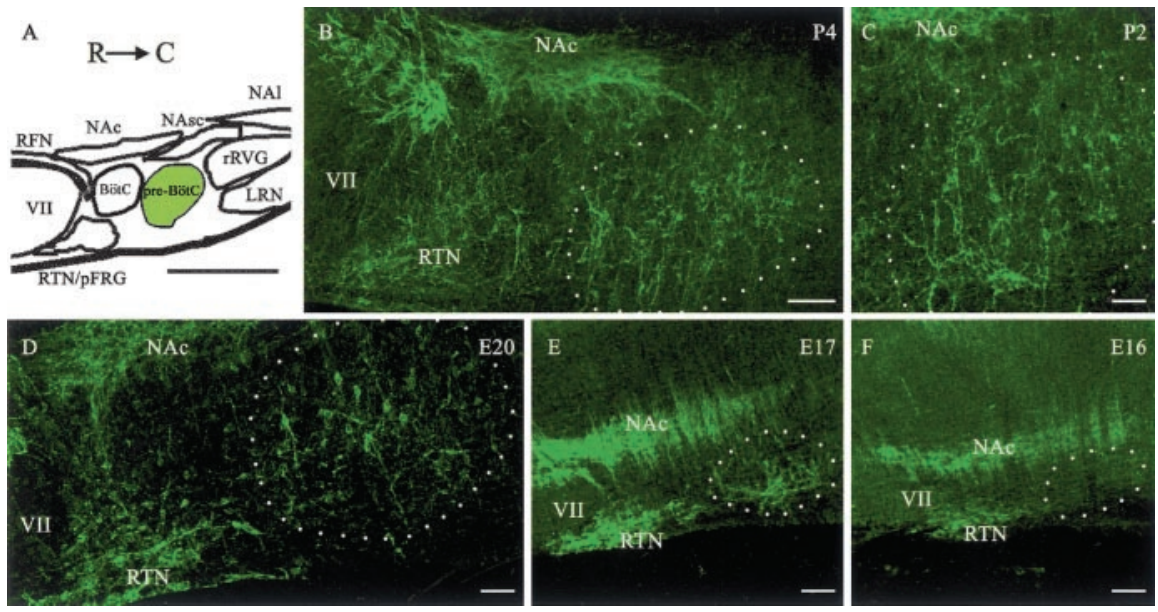

Figure 1. Rostrocaudal distribution of NK1R-positive neurons within the ventrolateral medulla of perinatal rats. $A$, Primary structures in the ventrolateral medulla, which include VII, NAc, NAsc, nucleus ambiguus, loose formation (NAI), RTN, pFRG, RFN, Bötzinger complex (Bötz), pre-BötC, rVRG, and LRN. R, Rostral; C, caudal. B-F, Representative examples of immunolabeling for NK1R (green) in P4, P2, E20, E17, and E16 sagittal sections of the ventrolateral medulla. The dashed circle demarcates the approximate area of the pre-BötC. Scale bars: $A, 700 \mu \mathrm{m} ; B, E, F, 100 \mu \mathrm{m} ; C, D, 50 \mu \mathrm{m}$.

touching its ventral border, were considered during the counting process. NK1R-positive neurons with or without BrdU-labeled nuclei were counted, and statistical analysis of the results was performed as reported in Figure 6.

Measurements of cell area were obtained from confocal acquired images using LSM 510 software. NK1R-, SST-, and BrdU-positive cells were identified in the $z$-stack planes, and the area was calculated for each cell. Statistics were calculated using Prism 3.0 software (GraphPad Inc., San Diego, CA). Values given are means and SE, and the raw data and medians are plotted in Figures 2, 7, and 10.

In vitro preparations. Immediately on delivery, the neuraxis was isolated from embryonic rats, leaving the diaphragm attached for EMG recordings (Smith et al., 1991). To prepare medullary slice preparations, the brain stem-spinal cord was pinned down, ventral surface-upward, on a paraffin-coated block. The block was mounted in the vise of a Leica VT1000S vibratome. A single transverse slice containing the pre-BötC and more caudal reticular formation regions was then cut, transferred to a recording chamber, and pinned down onto a Sylgard elastomer. The cytoarchitecture was observed under infrared differential interference contrast (IR-DIC) optics (Leica Axioskop) to confirm that a similar rostral level of the medulla was recorded from at each age. The medullary slice was continuously perfused $(5 \mathrm{ml} / \mathrm{min})$ at $27 \pm 1^{\circ} \mathrm{C}$ (chamber volume, $1.5 \mathrm{ml}$ ) with a solution containing (in $\mathrm{mM}$ ): $128 \mathrm{NaCl}, 9.0 \mathrm{KCl}, 1.5$ $\mathrm{CaCl}_{2}, 1.0 \mathrm{MgSO}_{4}, 24 \mathrm{NaHCO}_{3}, 0.5 \mathrm{NaH}_{2} \mathrm{PO}_{4}$, and $30 \mathrm{D}$-glucose equilibrated with $95 \% \mathrm{O}_{2}$ and $5 \% \mathrm{CO}_{2}$.

Recording and analysis of inspiratory discharge. Recordings of diaphragm EMG or hypoglossal (XII) nerve roots were made with suction electrodes. Furthermore, suction electrodes were placed into XII nuclei and the pre-BötC under visual guidance using an IR-DIC microscope to record extracellular neuronal population discharge from the medullary slice. Signals were amplified, rectified, low-pass-filtered, and recorded using an analog-to-digital converter (Digidata 1200; Axon Instruments, Foster City, CA) and data acquisition software (Axoscope). The effects of adding SP (100 nM) were evaluated using E17 preparations. Values given are means, SD, and coefficients of variability (SD/mean). Statistical significance was tested using paired difference Student's $t$ test; significance was accepted at $p<0.05$.

\section{Results}

\section{NK1R immunolabeling in the newborn medulla}

The initial stage of the study examined the spatiotemporal profile of NK1R expression in the medulla of newborn rats (P2 and P4; $n=4$ for each age). Sagittal sectioning provided a clear view of NK1R labeling within key respiratory nuclei located along the rostrocaudal axis of the ventrolateral medulla (Fig. 1). Figure 1, $B$ and $C$, shows examples of NK1R labeling in sagittal sections of the ventrolateral medulla at $\mathrm{P} 4$ and $\mathrm{P} 2$, respectively. The areas of particular interest, from rostral to caudal levels, included the facial nucleus, retrotrapezoid nucleus (RTN), parafacial respiratory group (pFRG), retrofacial nucleus (RFN), compact division of the nucleus ambiguus (NAc), pre-BötC, semicompact division of the nucleus ambiguus (NAsc), and rostral ventral respiratory group (rVRG). NK1R was present in several brainstem nuclei during the first postnatal week. Intense immunoreactivity was present in the cell bodies of the NAc, RTN, pFRG, and RFN, whereas the NAsc showed weaker immunostaining. In the pre-BötC, cell bodies were strongly immunolabeled for NK1R at all postnatal ages studied. NK1R-positive neurons within the pre-BötC area were generally small with prominent dendrites orientated in the dorsoventral axis $\left(\mathrm{P} 2,149.7 \pm 8.5 \mu \mathrm{m}^{2}\right.$; P4, $183.9 \pm 8.4 \mu \mathrm{m}^{2}$ ). Figure 2 shows the population data for NK1Rpositive neuronal areas at all perinatal ages studied. The rostrocaudal extents of the pre-BötC were $\sim 350-700$ and $400-800 \mu \mathrm{m}$ caudal to the facial nucleus at P2 and P4, respectively. Neither the Bötzinger region, located rostral to the pre-BötC, nor the rVRG was immunoreactive for NK1R.

Although the ventrolateral medulla was the primary focus of the study, we did note the pattern of NK1R labeling within other medullary structures (Fig. 3). Intense labeling for NK1R was present on processes extending out of the dorsal side of the hypoglossal nucleus and along the midline. Several cell bodies and processes in the solitary tract nucleus (STN) were strongly immunoreactive for NK1R, and sparse numbers of immunolabeled cells were present in the spinal trigeminal nucleus as well as large cells in the gigantocellular reticular nucleus and the paramedian reticular nucleus. The neuropil in the inferior olive was lightly immunoreactive, whereas intense cellular labeling was present at the level of the raphe pallidus.

\section{NK1R immunolabeling in the prenatal medulla}

The second stage of our study examined NK1R expression in the medulla during prenatal development (Figs. $1 D-F, 2)$. At E15 $(n=19)$, strong NK1R expression was evident in the NA, the solitary tract, midline structures, and spinal trigeminal nuclei (data not shown). Weak staining was also present in the RTN, pFRG, medial neuroepithelium, and rhombic lip. The intensity of NK1R immunolabeling within medullary nuclei expressing NK1R at E15 increased markedly at E16 $(n=15)$ and older embryonic ages. There was no obvious NK1R labeling in the region of the pre-BötC at E15. By E16, there was weak neuropilar staining within the pre-BötC. It was not until E17 $(n=14)$ that NK1R expression was clearly detectable in cell bodies within the pre-BötC. Most NK1R-positive neurons at E17 were small $\left(99.9 \pm 2.2 \mu \mathrm{m}^{2}\right)$. From ages E18 to E20 $(n=12)$, the intensity of the NK1R immunostaining and cell area increased (E18, $103.2 \pm$ $3.13 \mu \mathrm{m}^{2}$; E20, $132.9 \pm 4.1 \mu \mathrm{m}^{2}$ ). The rostrocaudal extents of the pre-BötC were $\sim 300-550$ and $325-650 \mu \mathrm{m}$ caudal to the facial nucleus at E17 and E18, respectively. 


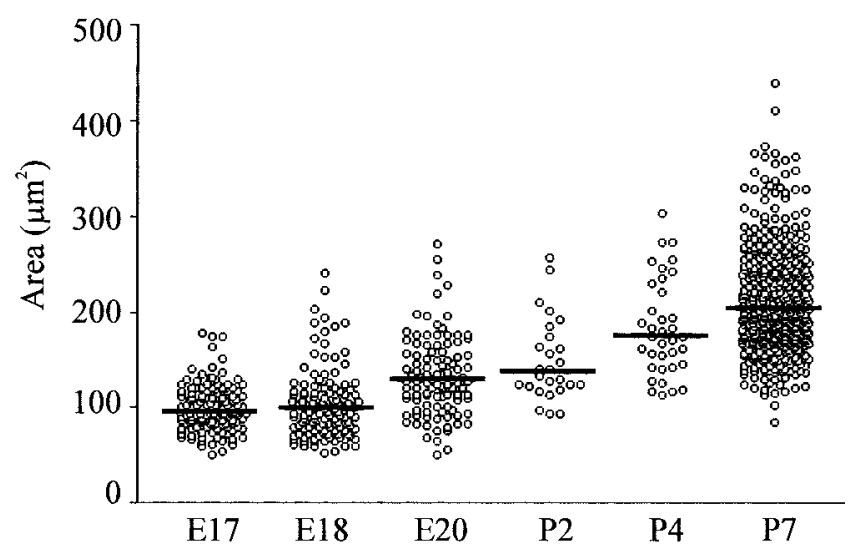

Figure 2. Size distribution of NK1R-positive neurons located in the pre-BötC at different perinatal ages. A plot of individual somal areas of NK1R-positive cells located within the preBötC is shown. Horizontal lines show the median of the population data for each age.

\section{NK1R and ChAT expression in the prenatal ventrolateral medulla}

Past studies of the adult medulla had demonstrated that ChATpositive neurons in the ventrolateral medulla also express NK1R. Thus, it was important to differentiate between those neurons, which include motoneurons from the pharyngeal branch of the vagus nerve (Bieger and Hopkins, 1987), and non-ChATexpressing pre-BötC neurons. We performed double labeling for ChAT and NK1R in transverse sections of medullas isolated from ages E16 to E20 ( $n=4-7$ for each age; Fig. 4). Colocalization for NK1R and ChAT expression was evident at the level of the NAc at every perinatal age investigated. No motoneurons in the external formation of the NA expressed NK1R. As mentioned above, there was no clear staining for NK1R in the pre-BötC at E16, although motoneurons in the compact formation of the NA were intensely stained for NK1R and ChAT. At this early stage, the motoneurons were located somewhat ventral to the position observed in the newborn and adult. Furthermore, several NK1R- and ChATexpressing motoneurons extended processes toward the ventral surface of the medulla. The first clear indication of NK1R immunoreactivity in non-ChAT-expressing neurons within the region of the pre-BötC was evident at E17 and increased through to E20 (Fig. 4).

\section{Birth dating of medullary neurons}

Cells undergoing their final stages of division before migration from the ventricular zone to medullary nuclei were labeled by exposure to BrdU at different gestational ages (E10.5-E16.5). BrdU is a thymidine analog used to determine cell proliferation, migration, and genesis of cells in different nervous structures (del Rio and Soriano, 1989; Nowakowski et al., 1989). BrdU is incorporated in actively dividing cells when they enter the S-phase of the cell cycle and can be detected by means of a BrdU antibody. We performed double labeling for BrdU and NK1R on transverse sections from P7 rats $(n=40$; Fig. 5$)$ to identify NK1R-positive neurons born at different developmental times. At P7, NK1Rpositive neurons located in the pre-BötC were spherical, fusiform, or irregularly shaped $\left(213.6 \pm 2.9 \mu \mathrm{m}^{2}\right)$.

Presumptive NA motoneurons that were NK1R-positive incorporated a high amount of BrdU after injections on E10.5 and to a lesser extent after injections on E11.5. Other structures labeled intensely after E10.5 BrdU injections included regions containing VII, X, and XII motoneurons. After injection on E11.5,
BrdU-positive nuclei were intensely labeled in the RTN, pFRG, STN, and Sp5, gigantocellular (Gi), intermediate reticular (IRt), and vestibular (Ve) nuclei. Only a few nuclei were detected in the VRG. BrdU injections on E12.5 labeled several nuclei throughout the medulla, including the STN and Sp5, IRt, parvocellular reticular (PCRt), and raphe nuclei. Peak BrdU incorporation within NK1R-positive pre-BötC neurons resulted from injections on E12.5 and E13.5 (Fig. 6). Cell counts of NK1R-positive neurons within the pre-BötC demonstrated that peak birth dates at E12.5 $(33.1 \pm 5.3 \%)$ and E13.5 (38.0 $\pm 6.1 \%)$. A small population of NK1R-expressing neurons in the region of the pre-BötC was born before $(\mathrm{E} 11.5,10.1 \pm 4.6 \%)$ and after $(\mathrm{E} 14.5,2.0 \pm 1.6 \%)$ that period. Other nuclei labeled by injections on E13.5 included the rVRG, Gi, PCRt, Sp5, Ve, area postrema, external caudate, and inferior olive nuclei. Injections on E15.5 and E16.5 labeled a small number of NK1R-negative cells distributed along the dorsal and lateral medulla.

Cell size distribution analysis of NK1R-positive neurons generated at different times (Fig. 7) indicated that larger neurons were generated earlier $\left(\mathrm{E} 11.5,269.8 \pm 15.21 \mu \mathrm{m}^{2}\right)$, whereas most of the small neurons were labeled with BrdU during the peak generation of pre-BötC neurons (E12.5, $211.5 \pm 5.8 \mu \mathrm{m}^{2}$; E13.5, $\left.205 \pm 7.2 \mu \mathrm{m}^{2}\right)$.

BrdU, NK1R, and ChAT in the prenatal ventrolateral medulla To further characterize the birth date and timing of migration of NK1R-positive neurons in the pre-BötC, we injected single pulses of BrdU on E10.5, E11.5, E12.5, and E13.5 and collected the embryos at different gestational ages (E15-E18). Triple labeling for NK1R, BrdU, and ChAT allowed us to identify different neuronal populations in the ventrolateral medulla (Fig. 8).

Figure $8 \mathrm{~A}-\mathrm{C}$ illustrates the distribution of cells within the E15 ventrolateral medulla $(n=12)$ labeled for BrdU at ages E10.5, E11.5, and E12.5, respectively. There was a high concentration of ChAT- and NK1R-positive neurons in the NA and facial nucleus at E15 that were born at E10.5. Furthermore, some ChATpositive, NK1R-negative neurons born at E10.5 appeared along their putative migratory pathway from the dorsal neuroepithelium to the ventrolateral medulla (Fig. 8A). Cells born at E11.5 were distributed more diffusely in the ventrolateral medulla at E15, with a pronounced cluster in the ventrolateral medulla spanning rostrocaudally from the facial nucleus to the rVRG. Injection of BrdU on E12.5 labeled a large population of cells dorsal to the NA (Fig. 8C).

Figure $8 D-F$ illustrates the distribution of cells within the E16 ventrolateral medulla $(n=14)$ labeled for BrdU at ages E11.5, E12.5, and E13.5, respectively. Cells born at E11.5, E12.5, and E13.5 were distributed both dorsally and ventrally to the NA at E16. There were very few BrdU-positive cells among the NK1Rand ChAT-positive neurons.

Figure $8, G$ and $H$, illustrates the distribution of cells within the E17 ventrolateral medulla $(n=9)$ labeled for BrdU at ages E12.5 and E13.5, respectively. Figure $8 I$ illustrates the distribution of cells labeled for BrdU at E13.5 within the E18 ventrolateral medulla $(n=3)$. By E17, a large number of neurons within the pre-BötC area expressed NK1R. Many of the NK1R-positive neurons found within the pre-BötC at E17 were born at E12.5 (Fig. $8 G)$. In contrast, most NK1R-positive neurons within the preBötC born on E13.5 were not detected in the area until E18 (Figs. $8 H-I)$. 

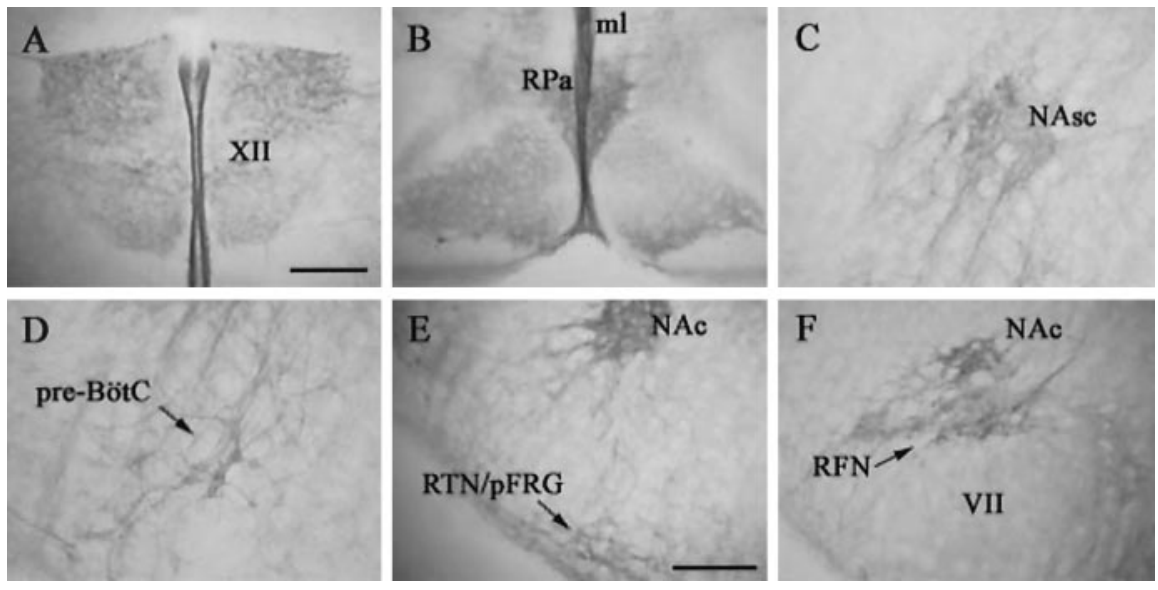

Figure 3. NK1R expression in medullary structures of $\mathrm{P} 2$ rats. Photomicrographs from transverse medullary sections illustrate the pattern of NK1R labeling in various medullary nuclei, including XII, midline (ml), raphe pallidus (Rpa), NAc, NAsc, pre-BötC, RTN, pFRG, and RFN. Scale bars: $A-D, 200 \mu \mathrm{m} ; E, F, 200 \mu \mathrm{m}$.
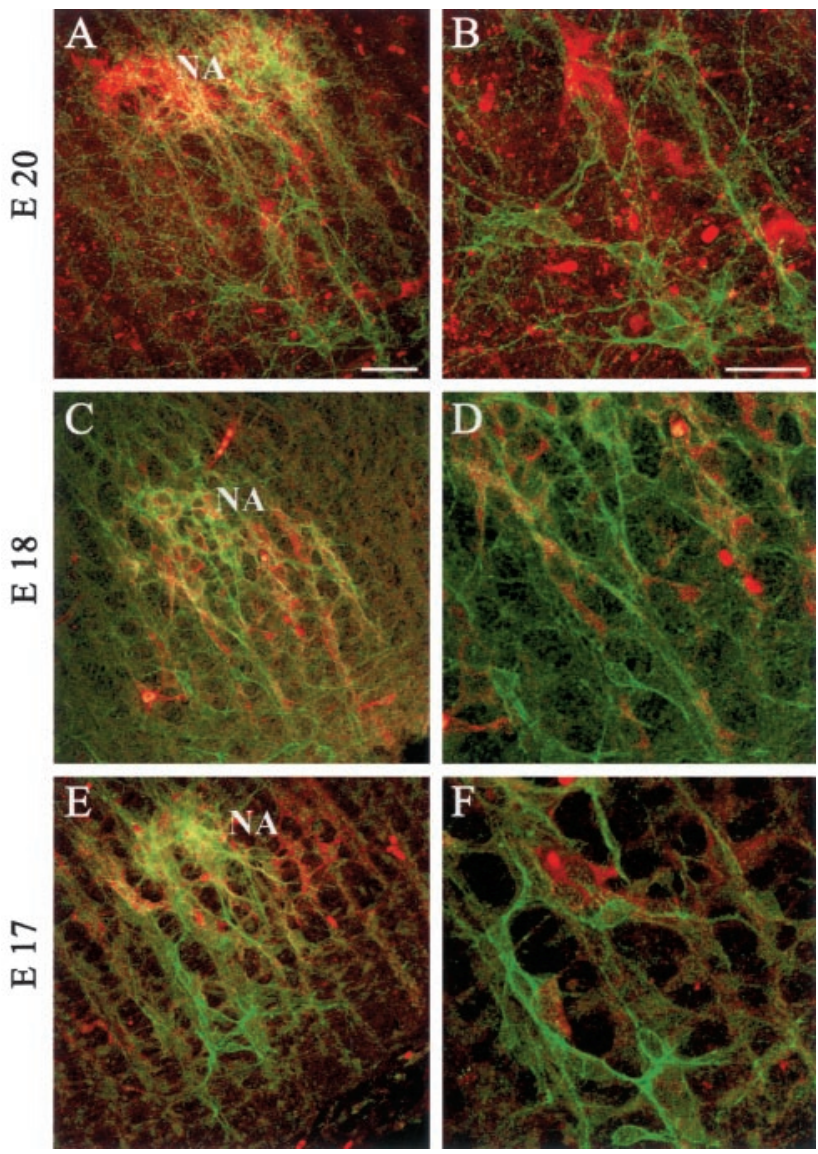

Figure 4. Colocalization of NK1R and ChAT is prominent in the NAsc but mostly absent within the pre-BötC of prenatal rats. Immunoreactivity for ChAT (red) and NK1R (green) is shown in transverse sections at the level of the NAsc and pre-BötC at E20 $(A, B), \mathrm{E} 18(C, D)$, and E17 $(E, F)$. $B, D, F$, Higher-magnification views of the pre-BötC from $A, C, D$, respectively. Double-labeled cells appear yellow. Scale bars, $50 \mu \mathrm{m}$.

\section{Somatostatin expression in the perinatal ventrolateral medulla}

We analyzed SST immunolabeling in the developing ventrolateral medulla during embryonic and perinatal development as a further means of examining pre-BötC ontogenesis (Fig. $9 ; n=$ 21). In the perinatal medulla, SST was present in a high number of neurons located dorsal and ventral to the NA. Intense labeling for SST was detected within the VRG, including some, but not all, NK1R-positive neurons in the pre-BötC area. In postnatal rats, expression for SST was particularly intense at the level of the pre-BötC, at which several neurons expressed both NK1R and SST. On P7, 33.3\% of SST-positive neurons expressed NK1R, and $34.4 \%$ of NK1Rpositive neurons colocalized with SST $(n=65)$. The average cell area of SSTpositive neurons was $176.4 \pm 5.13 \mu \mathrm{m}^{2}$ (Fig. 10). It should be noted that we detected a high number of SSTimmunoreactive cells within the pre-BötC and in the adjacent respiratory areas (both the Bötzinger complex and rVRG) that did not coexpress NK1R. Neurons expressing high levels of NK1R located in the RTN, pFRG, NAc, and RFN did not coexpress SST at any age examined (E15-P4). SST expression in the ventrolateral medulla increased during the developmental period studied. At E15, expression of SST was strongly detectable in tangential fibers, whereas neuronal cell bodies within the ventrolateral medulla expressed a very low amount of the neuropeptide. By E17, when NK1R-positive neurons within the pre-BötC area were detectable, expression of SST was present in both NK1R-positive and -negative neurons. On E18 and E20, respectively, 38.9\% $(n=117)$ and 38.8\% $(n=$ 225) of SST-positive neurons expressed NK1R, whereas 35.9 and $52.0 \%$ of NK1R-positive neurons coexpressed SST. Doublelabeling experiments for ChAT and SST showed a number of motoneurons in the rostral part of the external formation of the NA that also expressed SST. Few scattered motoneurons in the facial nucleus were positive for SST.

The mean cell area of SST-positive neurons in the pre-BötC was slightly less on average than in NK1R-positive neurons. The average cell area was $94.07 \pm 5.7$ and $97.9 \pm 3.8 \mu \mathrm{m}^{2}$ on E17 and E18, respectively. The average area of SST-positive neurons increased to $115.9 \pm 4.7 \mu \mathrm{m}^{2}$ on E20.

\section{Electrophysiological recordings from perinatal medullary slice preparations}

To determine when inspiratory motor discharge commences in the medulla, suction electrode recordings were made from the regions of the pre-BötC and hypoglossal motor pool from ages E15 to P2 (Fig. 11, Table 1). A clear inspiratory discharge was observed in all medullary preparations isolated at ages E17 and beyond. An apparent inspiratory rhythmic discharge recorded from both the pre-BötC and hypoglossal motor pool was present in 5 of 10 medullary slice preparations isolated at E16.5. At earlier developmental ages, we occasionally observed a robust rhythmic discharge with characteristics different from inspiratory bursts. The burst duration $(\sim 2 \mathrm{sec})$ and interburst interval $(\sim 40 \mathrm{sec})$ were significantly longer than inspiratory rhythmic motor patterns. This nonrespiratory rhythmic activity was very characteristic of the spontaneous motor discharge generated at multiple sites along the embryonic rat neuraxis (Ren and Greer, 2003) (for review, see Ben-Ari, 2001). With advancing embryonic age, there were significant increases in the frequency and amplitude of inspiratory motor discharge. Furthermore, the delay between the onset of XII motor discharge and pre-BötC population discharge decreased. 

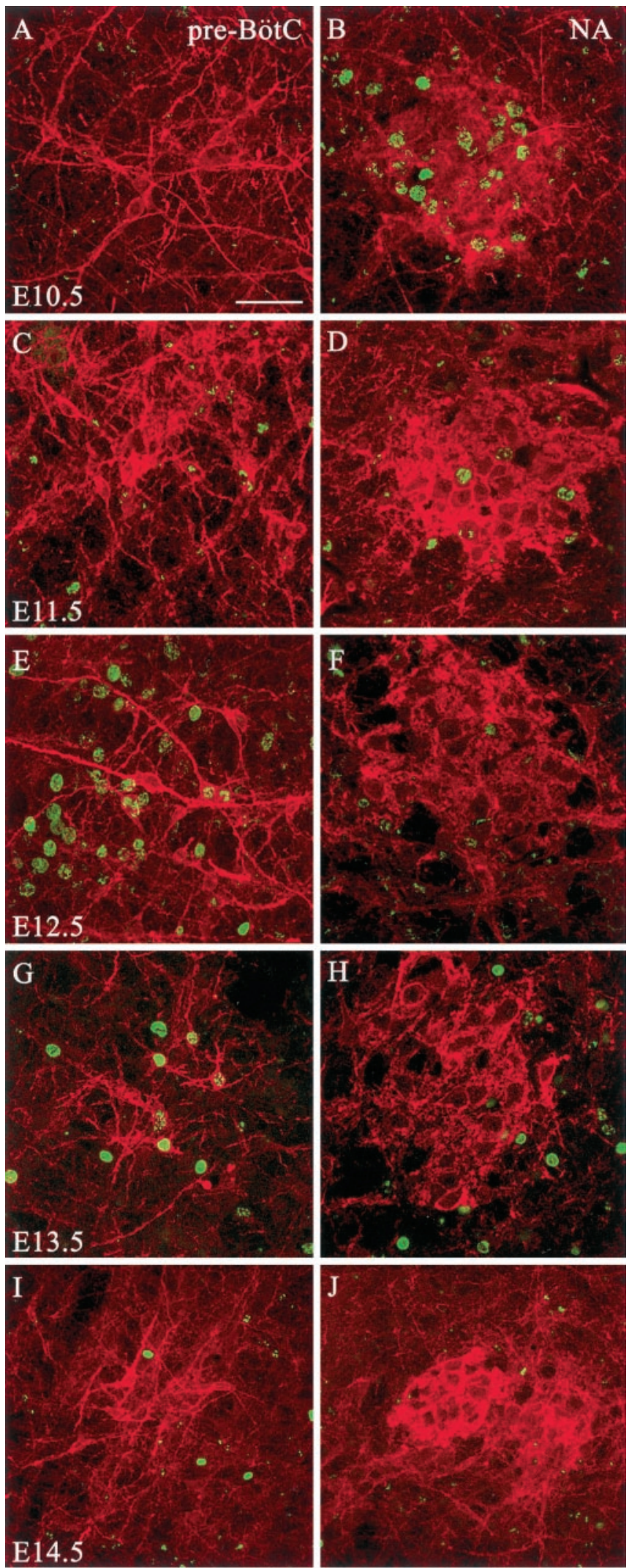

Figure 5. NK1R-positive neurons within the pre-BötC are born $\sim 2 \mathrm{~d}$ later than those within the NAsc. $A, C, E, G, I$, Representative immunolabeling for NK1R (red) and BrdU (green) within the pre-BötC of transverse sections of $P 7$ rats. $B, D, F, H, J$, Representative immunolabeling for NK1R (red) and BrdU (green) within the NAsc of transverse sections of P7 rats. The BrdU was injected on different days ranging from E10.5 to E14.5. Scale bar, $50 \mu \mathrm{m}$.

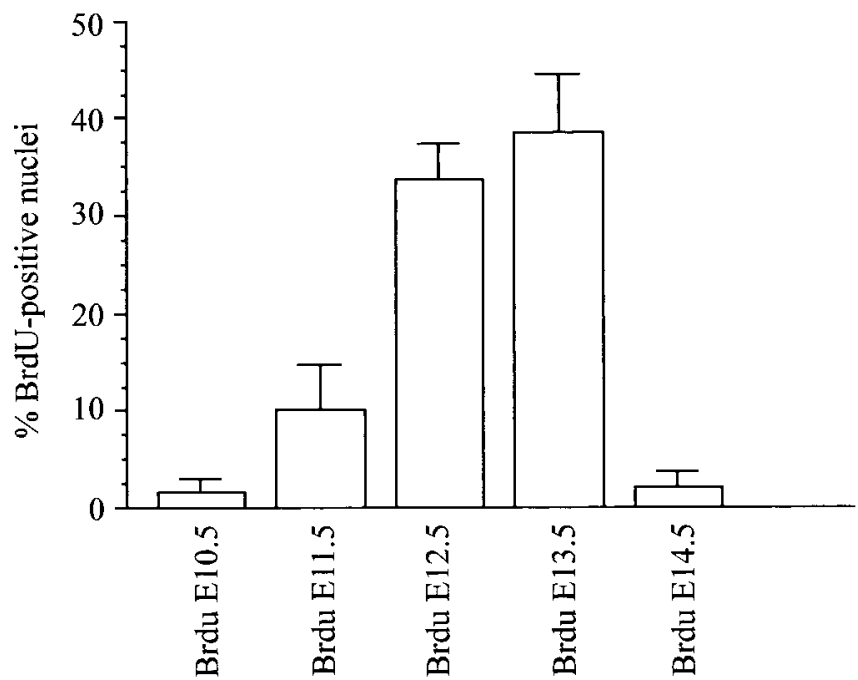

Figure 6. Most NK1R-positive neurons within the pre-BötC are born on E12.5 and E13.5. The plot shows the percentages of NK1R-positive neurons in the region of the pre-BötC that have BrdU-positive nuclei after injections on different days ranging from E10.5 to E14.5.

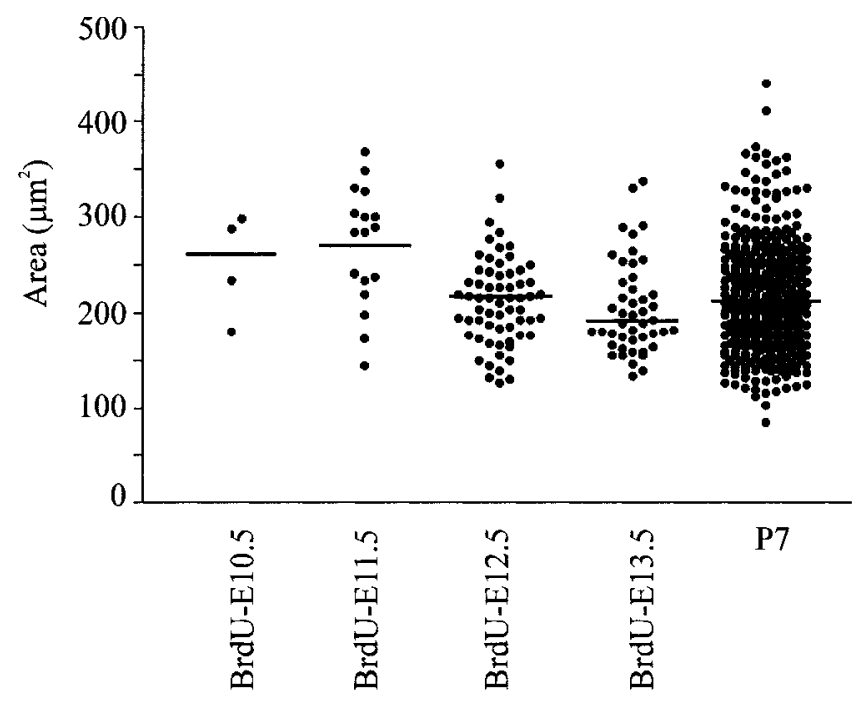

Figure 7. Size of NK1R-positive neurons born on E12.5 and E13.5 located in the pre-BötC The plot shows individual somal areas of cells located within the pre-BötC that are labeled for both NK1R and BrdU on P7. The BrdU was injected on different days ranging from E10.5 to E13.5. Horizontal lines show the median of the population data for each age.

Given that the immunohistochemical data had demonstrated the inception of NK1R expression within pre-BötC neurons at E17, we examined the effects of endogenously applied SP. As shown in Figure 12A, there was a pronounced increase in the respiratory rhythm frequency generated by the medullary slice with application of $100 \mathrm{nM}$ SP to the bathing medium (360 \pm $78 \%$ of control; $n=3)$. These results differ from those reported in an earlier study (Ptak et al., 1999) reporting no modulatory action of applied SP on the respiratory rhythm before E20 in the brainstem-spinal cord preparation. To determine whether the difference could be accounted for by the different types of in vitro preparations, we repeated the SP application to the medium bathing brainstem-spinal cord preparations with the diaphragm muscle attached. As shown in Figure $12 B$, there was also marked stimulation of respiratory frequency at E17 (419 $\pm 92 \%$ of control; $n=3$ ). 

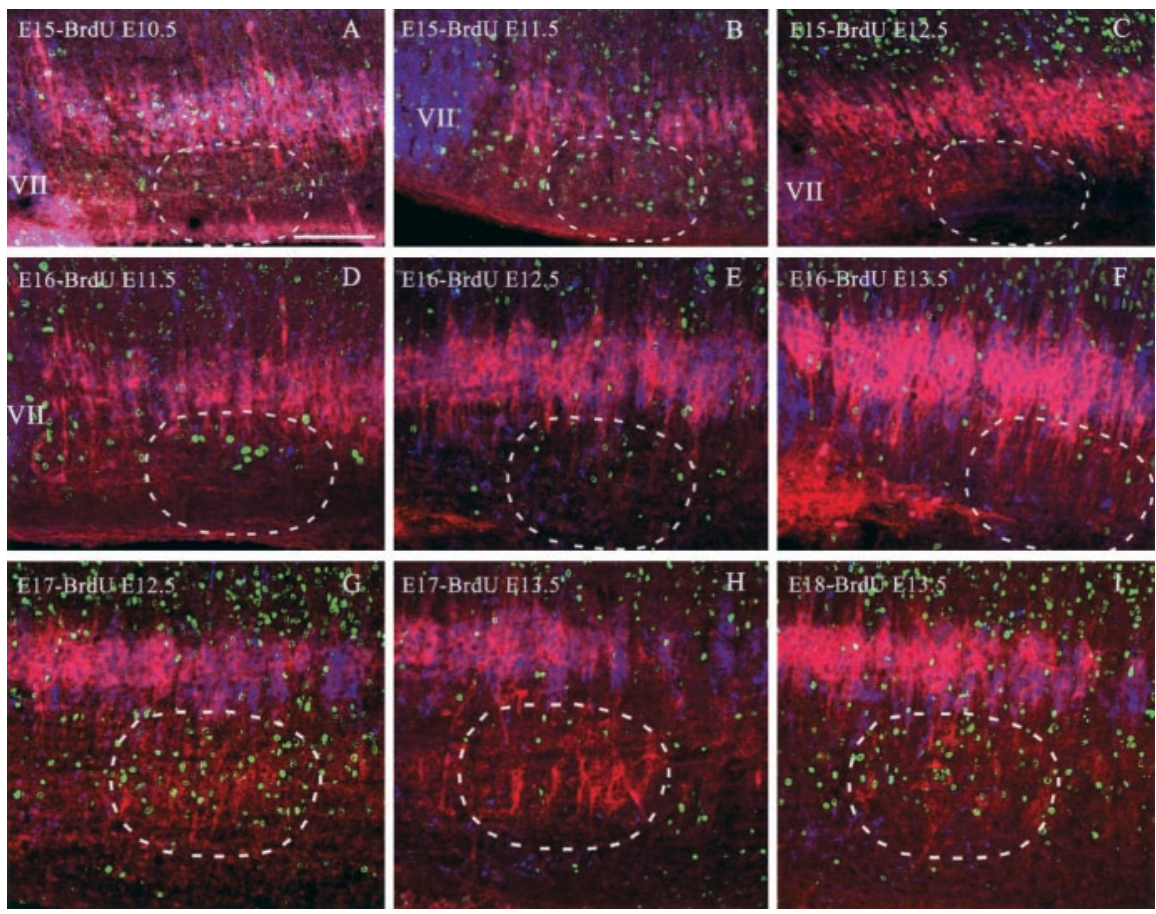

Figure 8. Spatiotemporal distribution of the birth dates of NK1R- and ChAT-positive neurons in the ventrolateral medulla of prenatal rats (E15-E18). Sagittal sections were immunolabeled for NK1R (red), ChAT (blue), and BrdU (green). Cells expressing both NK1R and ChAT appear magenta. A-C, Labeling patterns atE15 after BrdU injections on E10.5, E11.5, and E12.5, respectively. $D-F$, Labeling patterns at E16 after BrdU injections on E11.5, E12.5, and E13.5, respectively. $G, H$, Labeling patterns at E17 after BrdU injections on E12.5 and E13.5, respectively. I, Labeling pattern at E18 after BrdU injections on E13.5. The dashed circles demarcate the approximate area of the pre-BötC. Scale bar, $100 \mu \mathrm{m}$.
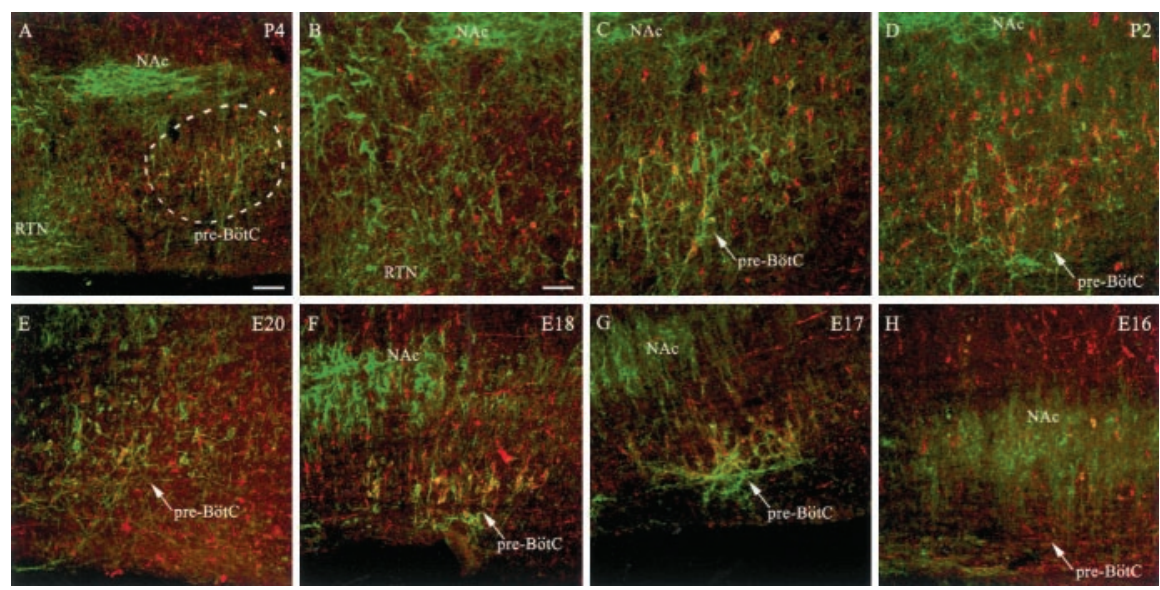

Figure 9. A subpopulation of neurons within the pre-BötC of perinatal rats expresses both SST and NK1R. A, Representative examples of immunolabeling for NK1R (green) and SST (red) in a sagittal section of a P4 ventrolateral medulla. Double-labeled neurons appear yellow. $B$, Higher-magnification view of $A$ showing the area immediately rostral to the facial nucleus. $C$, Highermagnification view of $A$ showing the pre-BötC region. $D-H$, Region of the pre-BötC in transverse sections from animals aged $P 2$, $\mathrm{E} 20, \mathrm{E} 18, \mathrm{E} 17$, and E16. The dashed circle demarcates the approximate area of the pre-BötC. Scale bars: $A, 700 \mu \mathrm{m} ; B, E, F, 100 \mu \mathrm{m}$; $C, D, 50 \mu \mathrm{m}$.

\section{Discussion}

The immunohistochemical and electrophysiological data from this study indicate that a key component of the respiratory rhythm-generating center, the pre-BötC, forms and becomes functional at $\sim$ E16.5-E17 in the rat. Furthermore, the NK1Rpositive neurons that populate the area of the pre-BötC during late E16-E18 are born at E12.5-E13.5, approximately $2 \mathrm{~d}$ later than adjacent NK1R-positive neurons in the ventrolateral medulla.

\section{Anatomical and}

immunohistochemical data

The NK1R has proven to be a valuable marker for identifying neurons within the pre-BötC (Gray et al., 1999, 2001; Guyenet and Wang, 2001; Liu et al., 2001; Wang et al., 2001; Guyenet et al., 2002). This is the first use of NK1R expression to discern the ontogenesis of the pre-BötC throughout the perinatal period. Postnatally, the pattern of NK1R expression in the medulla found in the current study is similar to that reported for the adult. The exceptions are that we did not see significant labeling in the postnatal rVRG (Makeham et al., 2001). We also did not observe a marked decrease in NK1R expression at P4 in the neonate, as reported previously (Liu and Wong-Riley, 2002). SST expression has also been proposed as a marker for preBötC neurons in the adult rat (Stornetta et al., 2003). Most SST-positive, fusiformshaped neurons within the adult pre-BötC are NK1R-positive and glutamatergic and project to the contralateral pre-BötC and thus fit the profile of respiratory rhythmogenic neurons. We also found clear SST expression in small fusiform-shaped neurons within the perinatal pre-BötC. However, the degree of overlap between SST and NK1R immunoreactivy within preBötC neurons was not as prominent as in the adult. Furthermore, the distribution of SST immunolabeling was more extensive in regions adjacent to the pre-BötC. These differences could reflect age-dependent changes in expression, the immunohistochemical methods used, or both.

The NK1R and SST immunohistochemical data provide for a general view of the anatomical inception of the pre-BötC. However, although the pre-BötC contains neurons involved directly in respiratory rhythmogenesis, the population is not homogeneous. The region is thought to contain respiratory rhythmogenic pacemaker neurons and propriobulbar interneurons as well as nonrespiratory neurons. Furthermore, unlike in the adult, there is likely a significant population of bulbospinal neurons within the pre-BötC during the perinatal period (Ellenberger, 1999). Those neurons may have made up part of the small population of larger NK1R neurons found in the perinatal pre-BötC. It is currently not possible to delineate clearly between specific neuronal subtypes within the pre-BötC using molecular markers. Thus, a more precise understanding of the intricate details of pre-BötC internal ontogeny derived from immunohistochemical labeling is presently unobtainable.

The region located ventral to the facial nucleus is also of considerable importance to the neural control of respiration. We found intense NK1R labeling in that region from E16 onward. 


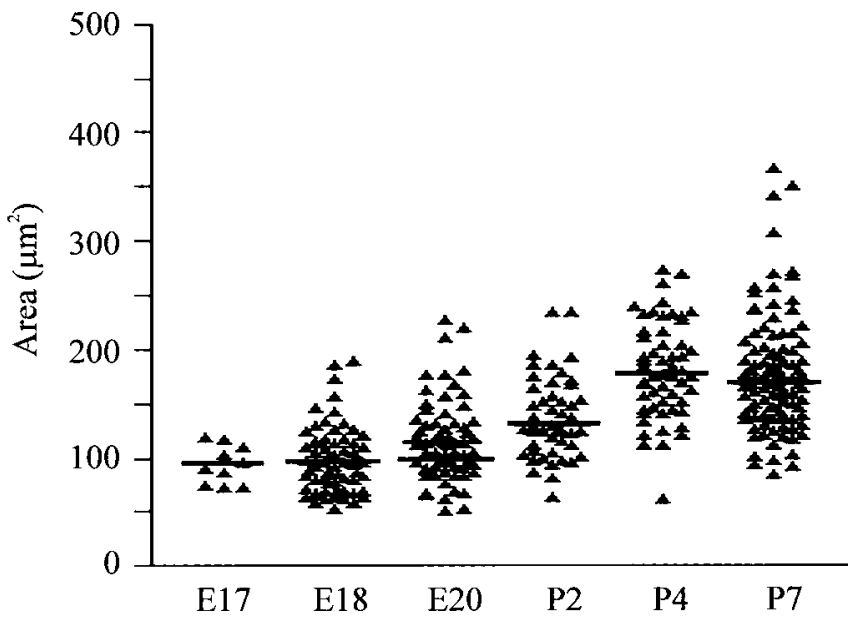

Figure 10. Size of SST-positive neurons within the pre-BötC. The plot shows of individual somal areas of SST-positive cells within the region of the pre-BötC at ages E17-P7. Horizontal lines show the median of the population data for each age.
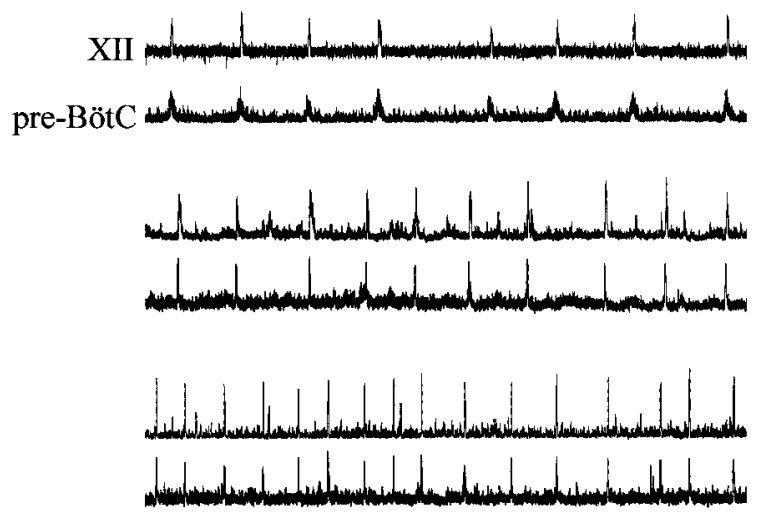

E17.5

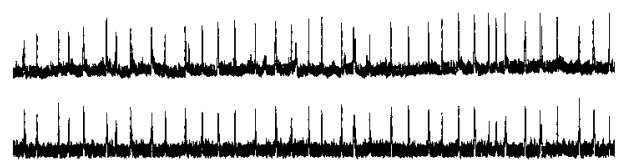

E20.5

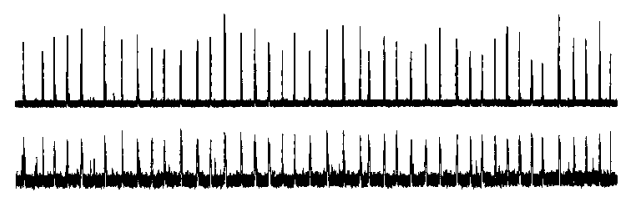

P2

$30 \mathrm{~s}$

Figure 11. Respiratory discharge patterns generated by perinatal medullary slice preparations. Sample rectified and integrated suction electrode recordings were made from neurons located within the XII nucleus and pre-BötC in medullary slice preparations isolated from E16.5-P2 perinatal rats.

This region includes the RTN, a cluster of NK1R-expressing neurons located ventral to the caudal portions of the facial nucleus that are chemosensitive and connected to respiratory nuclei in the dorsal and ventral medulla (Cream et al., 2002). Furthermore, recent evidence from optical recordings in newborn rat brainstem-spinal cord preparations suggests that neurons located ventral to the facial nucleus are involved in primary rhythm generation (Onimaru and Homma, 2003). The area has been referred to as the pFRG. The anatomical differentiation between the RTN
Table 1. Characterization of inspiratory bursts recorded from the pre-Bötzinger complex of rat medullary slice preparations isolated from perinatal rats

\begin{tabular}{|c|c|c|c|c|c|c|}
\hline Age & $n$ & Interval (sec) & Duration (sec) & $\begin{array}{l}\text { Amplitude } \\
\text { (mV) }\end{array}$ & $\begin{array}{l}\text { Coefficient of } \\
\text { variation of } \\
\text { burst interval }\end{array}$ & $\begin{array}{l}\text { Delay onset } \\
\text { (sec) }\end{array}$ \\
\hline 16.5 & 5 & $40 \pm 15^{*}$ & $1.3=$ & $6.1^{*}$ & 0.38 & 0.72 \\
\hline E17.5 & 8 & $24 \pm 8.7^{*}$ & $1.0 \pm 0.12^{*}$ & $19 \pm 5.7^{*}$ & 0.36 & $0.45 \pm 0.11^{*}$ \\
\hline E18.5 & 4 & $18 \pm 7.0^{*}$ & $0.9 \pm 0.13^{*}$ & $23 \pm 6.9$ & 0.39 & $0.34 \pm 0.12^{*}$ \\
\hline E19.5 & 4 & $14 \pm 5.1^{*}$ & $0.82 \pm 0.10$ & $21 \pm 7.9$ & 0.36 & $0.21 \pm 0.09^{*}$ \\
\hline E20.5 & 5 & $8.8 \pm 3.1$ & $0.74 \pm 0.09$ & $25 \pm 6.4$ & 0.35 & $0.12 \pm 0.07$ \\
\hline P2 & 5 & $6.7 \pm 2.3$ & $0.72 \pm 0.10$ & $27 \pm 7.2$ & 0.34 & $0.10 \pm 0.07$ \\
\hline
\end{tabular}

The mean interburst interval, duration, and amplitude of inspiratory bursts were calculated from suction electrode recordings of population inspiratory discharge within the region of the pre-BötC of medullary slice preparations from perinatal rats. The delay onset refers to the relative timing of the onset of inspiratory bursts recorded from the XII nucleus inspiratory discharge relative to that recorded within the pre-BötC. Results are means $\pm S D ; n$ is the number of preparations examined.

${ }^{*} p<0.05$ compared with P2, Student's $t$ test.
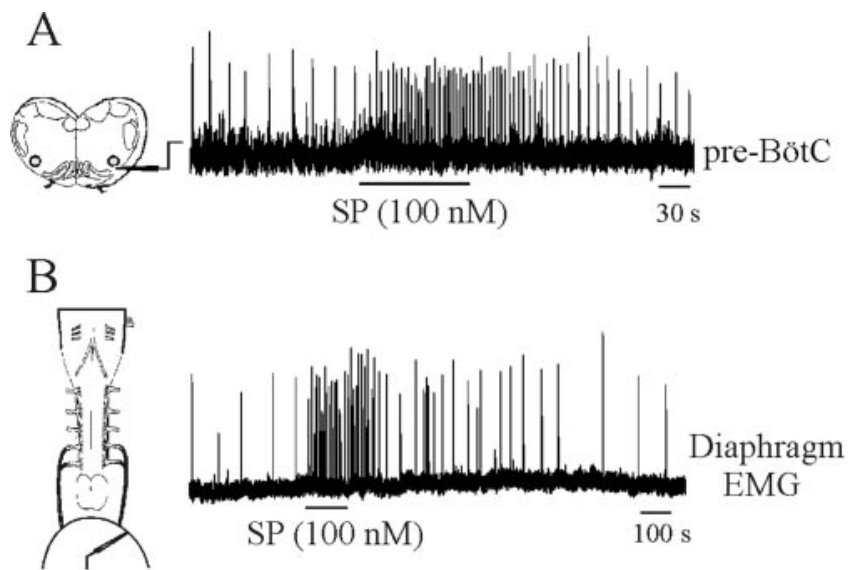

Figure 12. Substance $P$ causes a marked increase in the frequency of respiratory rhythm generated by $E 17$ in vitro preparations. $A$, Rectified and integrated suction electrode recordings made from the pre-BötC of an E17 medullary slice preparation. $B$, Rectified and integrated suction electrode recordings of diaphragm EMG activity from an E17 brainstem-spinal cord preparation. Substance $P$ was added to the perfusate during the period demarcated by the horizontal lines.

and pFRG is not clear because there appears to be some overlap, particularly in the caudal regions of the RTN. The pFRG contains a significant population of neurons that discharge during the preinspiratory phase (pre-I neurons). This class of neurons has been implicated in rhythm generation (Onimaru et al., 1989) and may be coupled to rhythm-generating neurons within the preBötC (Janczewski et al., 2002; Mellen et al., 2003). Interestingly, pre-I neurons respond to SP (Yamamoto et al., 1992) and thus may make up a portion of the neurons labeled in the current study. Single-cell recordings in conjunction with immunohistochemical labeling will be required to assess this clearly.

Birth dates of medullary neurons

Previous $\left[{ }^{3} \mathrm{H}\right]$ thymidine studies demonstrated that the genesis of medullary neurons in the rat occurs between E10 and E16 (Altman and Bayer, 1980; Bourrat and Sotelo, 1990, 1991; Phelps et al., 1990). To delineate specifically the birth date of NK1Rexpressing medullary neurons, we injected BrdU at various embryonic ages (E10-E16) in combination with NK1R and BrdU immunolabeling at P7 (i.e., when the pre-BötC is well demarcated by NK1R expression). Consistent with previous reports (Altman and Bayer, 1980; Phelps et al., 1990), we found that NA, XII, X, and XII motoneuron pools were the first nuclei generated 
in the medulla (peak birth at E10.5). The NK1R-positive neurons in the region of the RTN and pFRG had peak birth dates at E11.5. Most cells within the pre-BötC were born on E12.5 and E13.5. The only other NK1R-positive neurons born on a date similar to that of the pre-BötC neurons were located in the RFN, an area not associated with respiratory neuronal activity.

The second series of BrdU experiments performed used a combination of BrdU injections from E10 to E13 with immunolabeling for NK1R and BrdU at developmental periods E15-E18. This provided some insight regarding the time of settlement of NK1R-positive medullary neurons during embryonic development. We observed ChAT-positive neurons born on E10 and E11 that appeared to be migrating toward the NAc at E15. However, we did not see any evidence of NK1R expression in those neurons before their reaching the NAc. Nor did we observe any NK1R expression in any of the medullary neurons before their reaching nuclei from origins in the ventricular zone. Within the pre-BötC, a population of NK1R-expressing neurons born on E12 was evident at E17, and another population born on E13 was detected at E18. Our interpretations of these data are that (1) neurons within the medullary nuclei, including the pre-BötC, do not begin to express NK1R until their migratory phase is complete; and (2) the migration to the pre-BötC by the majority of NK1R-expressing neurons born on E12 and E13 is completed by E17 and E18, respectively.

\section{Electrophysiological recordings}

The recordings made from embryonic medullary slice preparations were critical for providing supportive data for the immunohistochemical and birth-dating studies. The lack of a well defined pre-BötC before E17 based on the NK1R and SST immunolabeling could reflect the fact that the critical neurons in the pre-BötC simply do not express those proteins at earlier ages. However, strong NK1R and SST labeling was observed in other medullary structures at earlier ages. The onset of rhythmic respiratory activity from the pre-BötC region at late E16 and E17 strongly supports the contention that the pre-BötC forms at that time. The increase in the amplitude and frequency of the rhythm at E18 is also consistent with an increase in NK1R-expressing neurons within the pre-BötC at that age. Our data demonstrating an increase in the amplitude and frequency of inspiratory discharge with advancing embryonic age are consistent with previous data derived from the brainstem-spinal cord preparation (Greer et al., 1992; DiPasquale et al., 1996; Onimaru and Homma, 2002; Viemari et al., 2003). The decrease in the delay between the onsets of XII motor discharge relative to that recorded in the pre-BötC could reflect increases in axonal diameter, myelination of projecting premotor axons, or both.

The marked increase in the frequency of respiratory discharge generated by medullary slice preparations at E17 in response to exogenous application of SP is informative in two regards. First, it indicates that functional NK1Rs are expressed by pre-BötC neurons regulating respiratory rhythmogenesis by E17. Second, it corroborates results from an earlier study of thyrotropinreleasing hormone (TRH) effects, demonstrating that the frequency of rhythmic respiratory discharge in the embryo can be increased toward neonatal levels by conditioning neuromodulation (Greer et al., 1996). Our studies did not assess the role of endogenously released SP in modulating respiratory rhythmogenesis. However, endogenous SP expression is present as early as E13 within the medulla (Horie et al., 2000). Thus, although SP is not necessary for generating a respiratory rhythm (Ptak et al., 2000), along with serotonin and TRH released from raphe neu- rons, it may play an important role in modulating perinatal respiratory drive.

Collectively, the data from in vitro nerve recordings and in vivo ultrasonography indicate that the neuronal network generating FBMs commences activity in a coordinated manner at $\sim$ E17 in the rat. Specifically, the following occurs: (1) the preBötC forms and commences respiratory rhythmogenesis; (2) bulbospinal projections extend to the cervical spinal cord (Lakke, 1997); and (3) diaphragm innervation and primary myogenesis are complete (Allan and Greer; 1997; Babiuk et al., 2003).

\section{Summary}

These novel data provide the necessary foundation for future work on the development of the pre-BötC, including the analyses of cell lineages and the transcriptional control of respiratory neuronal development and electrophysiological and pharmacological properties of the pre-BötC during the prenatal period. Furthermore, when extended to the murine model, this approach will allow for the determination of anatomical and functional anomalies within the pre-BötC of genetically engineered models with central apnea phenotypes (Shirasawa et al., 2000; Berry et al., 2003; Ren et al., 2003).

\section{References}

Allan DW, Greer JJ (1997) Embryogenesis of the phrenic nerve and diaphragm in the fetal rat. J Comp Neurol 382:459-468.

Altman J, Bayer SA (1980) Development of the brain stem in the rat. I. Thymidine-radiographic study of the time of origin of neurons of the lower medulla. J Comp Neurol 194:1-35.

Angulo y González AW (1932) The prenatal growth of the albino rat. Anat Rec 52:117-138.

Babiuk RP, Zhang W, Clugston R, Allan DW, Greer JJ (2003) Embryological origins and development of the rat diaphragm. J Comp Neurol 455:477-487.

Ben-Ari Y (2001) Developing networks play a similar melody. Trends Neurosci 24:353-360.

Berry GT, Wu S, Buccafusca R, Ren J, Gonzales L, Ballard P, Golden J, Greer JJ (2003) Lethal neonatal myo-inositol deficiency and central apnea in mice lacking the $\mathrm{Na}^{+}$/myo-inositol cotransporter (SLC5A3) gene. J Biol Chem 278:18297-18302.

Bieger D, Hopkins DA (1987) Viscerotopic representation of the upper alimentary tract in the medulla oblongata in the rat: the nucleus ambiguus. J Comp Neurol 262:546-562.

Bourrat F, Sotelo C (1990) Migratory pathways and selective aggregation of the lateral reticular neurons in the rat embryo: a horseradish peroxidase in vitro study, with special reference to migration patterns of the precerebellar nuclei. J Comp Neurol 294:1-13.

Bourrat F, Sotelo C (1991) Relationships between neuronal birthdates and cytoarchitecture in the rat inferior olivary complex. J Comp Neurol 313:509-521.

Cream C, Li A, Nattie E (2002) The retrotrapezoid nucleus (RTN): local cytoarchitecture and afferent connections. Respir Physiol Neurobiol 130:121-137.

del Rio JA, Soriano E (1989) Immunocytochemical detection of 5'bromodeoxyuridine incorporation in the central nervous system of the mouse. Brain Res Dev Brain Res 49:311-317.

DiPasquale E, Tell F, Monteau R, Hilaire G (1996) Perinatal developmental changes in respiratory activity of medullary and spinal neurons: an in vitro study on fetal and newborn rats. Brain Res Dev Brain Res 91:121-130.

Ellenberger HH (1999) Nucleus ambiguus and bulbospinal ventral respiratory group neurons in the neonatal rat. Brain Res Bull 50:1-13.

Feldman JL, Mitchell GS, Nattie EE (2003) Breathing: rhythmicity, plasticity, chemosensitivity. Annu Rev Neurosci 26:239-266.

Gray PA, Rekling JC, Bocchiaro CM, Feldman JL (1999) Modulation of respiratory frequency by peptidergic input to rhythmogenic neurons in the pre-Bötzinger complex. Science 286:1566-1568.

Gray PA, Janczewski WA, Mellen N, McCrimmon DR, Feldman JL (2001) 
Normal breathing requires pre-Bötzinger complex neurokinin-1 receptor-expressing neurons. Nat Neurosci 4:927-930.

Greer JJ, Smith JC, Feldman JL (1992) Generation of respiratory and locomotor patterns by an in vitro brainstem-spinal cord fetal rat preparation. J Neurophysiol 67:996-999.

Greer JJ, Al-Zubaidy ZA, Carter JE (1996) Thyrotropin releasing hormone (TRH) stimulates perinatal rat respiration in vitro. Am J Physiol 40:R1160-R1164.

Guyenet PG, Wang H (2001) Pre-Botzinger neurons with preinspiratory discharges "in vivo" express NK1 receptors in the rat. J Neurophysiol 86:438-446.

Guyenet PG, Sevigny CP, Weston MC, Stornetta RL (2002) Neurokinin-1 receptor-expressing cells of the ventral respiratory group are functionally heterogeneous and predominantly glutamatergic. J Neurosci 22:3806-3816.

Horie M, Miyashita T, Watabe K, Takeda Y, Kawamura K, Kawano H (2000) Immunohistochemical localization of Substance P receptors in the midline glia of the developing rat medulla oblongata with special reference to the formation of raphe nuclei. Brain Res Dev Brain Res 121:197-207.

Janczewski WA, Onimaru H, Homma I, Feldman JL (2002) Opioidresistant respiratory pathway from the preinspiratory neurones to abdominal muscles: in vivo and in vitro study in the newborn rat. J Physiol (Lond) 545:1017-1026.

Kobayashi K, Lemke RP, Greer JJ (2001) Ultrasound measurements of fetal breathing movements in the rat. J Appl Physiol 91:316-320.

Lakke EA (1997) The projections to the spinal cord of the rat during development: a timetable of descent. In: Advances in anatomy, embryology and cell biology, Vol 135, pp 1-43. New York: Springer.

Liu Q, Wong-Riley MT (2002) Postnatal expression of neurotransmitters, receptors, and cytochrome oxidase in the rat pre-Botzinger complex. J Appl Physiol 92:923-934.

Liu YY, Ju G, Wong-Riley MT (2001) Distribution and colocalization of neurotransmitters and receptors in the pre-Botzinger complex of rats. J Appl Physiol 91:1387-1395.

Makeham JM, Goodchild AK, Pilowsky PM (2001) NK1 receptor and the ventral medulla of the rat: bulbospinal and catecholaminergic neurons. NeuroReport 12:3663-3667.

Mantyh PW, Rogers SD, Honore P, Allen BJ, Ghilardi JR, Li J, Daughters RS, Lappi DA, Wiley RG, Simone DA (1997) Inhibition of hyperalgesia by ablation of lamina I spinal neurons expressing the substance P receptor. Science 278:275-279.

Mellen NM, Janczewski WA, Bocchiaro CM, Feldman JL (2003) Opioidinduced quantal slowing reveals dual networks for respiratory rhythm generation. Neuron 37:821-826.

Nowakowski RS, Lewin SB, Miller MW (1989) Bromodeoxyuridine immunohistochemical determination of the lengths of the cell cycle and the DNA-synthetic phase for an anatomically defined population. J Neurocytol 18:311-318.

Onimaru H, Homma I (2002) Development of the rat respiratory neuron network during the late fetal period. Neurosci Res 42:209-218.

Onimaru H, Homma I (2003) A novel functional neuron group for respiratory rhythm generation in the ventral medulla. J Neurosci 23:1478-1486.

Onimaru H, Arata A, Homma I (1989) Firing properties of respiratory rhythm generating neurons in the absence of synaptic transmission in rat medulla in vitro. Exp Brain Res 76:530-536.
Phelps PE, Brennan LA, Vaughn JE (1990) Generation patterns of immunocytochemically identified cholinergic neurons in rat brainstem. Brain Res Dev Brain Res 56:63-74.

Pilowsky PM, Feldman JL (2001) Identifying neurons in the pre-Bötzinger complex that generate respiratory rhythm: visualizing the ghost in the machine. J Comp Neurol 434:125-127.

Ptak K, DiPasquale E, Monteau R (1999) Substance P and central respiratory activity: a comparative in vitro study on foetal and newborn rat. Brain Res Dev Brain Res 114:217-227.

Ptak K, Hunt SP, Monteau R (2000) Substance P and central respiratory activity: a comparative in vitro study in NK1 receptor knockout and wildtype mice. Pflügers Arch 440:446-451.

Ramirez JM, Schwarzacher SW, Pierrefiche O, Olivera BM, Richter DW (1998) Selective lesioning of the cat pre-Bötzinger complex in vivo eliminates breathing but not gasping. J Physiol (Lond) 507:895-907.

Rekling JC, Feldman JL (1998) Pre-Bötzinger complex and pacemaker neurons: hypothesized site and kernel for respiratory rhythm generation. Annu Rev Physiol 60:385-405.

Ren J, Greer JJ (2003) Ontogeny of rhythmic motor patterns generated in the embryonic rat spinal cord. J Neurophysiol 89:1187-1195.

Ren J, Lee S, Pagliardini S, Gerard M, Stewart CL, Greer JJ, Wevrick R (2003) Absence of Ndn, encoding the Prader-Willi syndrome deleted gene necdin, results in congenital deficiency of central respiratory drive in neonatal mice. J Neurosci 23:1569-1573.

Shirasawa S, Arata A, Onimaru H, Roth KA, Brown GA, Horning S, Arata S, Okumura K, Sasazuki T, Korsmeyer SJ (2000) Rnx deficiency results in congenital central hypoventilation. Nat Genet 24:287-290.

Smith JC, Ellenberger HH, Ballanyi K, Richter DW, Feldman JL (1991) PreBötzinger complex: a brainstem region that may generate respiratory rhythm in mammals. Science 254:726-729.

Solomon IC, Edelman NH, Neubauer JA (1999) Patterns of phrenic motor output evoked by chemical stimulation of neurons located in the preBötzinger complex in vivo. J Neurophysiol 81:1150-1161.

Stornetta RL, Rosin DL, Wang H, Sevigny CP, Weston MC, Guyenet PG (2003) A group of glutamatergic interneurons expressing high levels of both neurokinin- 1 receptors and somatostatin identifies the region of the pre-Bötzinger complex. J Comp Neurol 455:499-512.

Sun Q, Goodchild AK, Pilowsky PM (2001) Firing patterns of pre-Bötzinger and Bötzinger neurons during hypocapnia in the adult rat. Brain Res 903:198-206

Viemari JC, Burnet H, Bevengut M, Hilaire G (2003) Perinatal maturation of the mouse respiratory rhythm-generator: in vivo and in vitro studies. Eur J Neurosci 17:1233-1244.

Vigna SR, Bowden JJ, McDonald DM, Fisher J, Okamoto A, McVey DC, Payan DG, Bunnett NW (1994) Characterization of antibodies to the rat substance $\mathrm{P}$ (NK-1) receptor and to a chimeric substance $\mathrm{P}$ receptor expressed in mammalian cells. J Neurosci 14:834-845.

Wang H, Stornetta RL, Rosin DL, Guyenet PG (2001) Neurokinin-1 receptor-immunoreactive neurons of the ventral respiratory group in the rat. J Comp Neurol 434:128-146.

Yamamoto Y, Onimaru H, Homma I (1992) Effect of substance P on respiratory rhythm and pre-inspiratory neurons in the ventrolateral structure of rostral medulla oblongata: an in vitro study. Brain Res 599:272-276. 